\title{
Prevention of falls and fall related injuries in older people in nursing homes and hospitals
}

\author{
H Handoll
}

Older people in residential care fall about three times as frequently as those living in the community. In part, this reflects higher incidences of risk factors for falls such as mobility limitations and dementia. Also, falls are an independent risk factor for admission to a nursing home. Falls in hospital settings are higher still: results from a Swedish study amounted to 6.2 falls per person year in a psychogeriatric ward, 3.4 falls per person year in a geriatric rehabilitation ward, and 1.1 falls per person year in a nursing home. ${ }^{1}$

Falls in nursing care facilities and hospitals often result in serious injuries and are associated with considerable mortality and morbidity. For example, rates of hip fracture as a result of falls in nursing care facilities have been estimated to be 10.5 times higher than in the community. ${ }^{2}$ Outcome too is poorer, as older people who fall while in hospital settings have significantly longer lengths of stay and are more likely to be discharged to a nursing home. ${ }^{3}$ Falls in these settings are thus a major public health issue and a wide range of interventions for preventing falls, often aimed at moderating risk factors, have been proposed and implemented. This points to the importance of the recent publication in the Cochrane Database of Systematic Reviews of a comprehensive systematic review that examines the effects of interventions for preventing falls in older people living in nursing care facilities and hospitals. ${ }^{4}$

\section{INTERVENTIONS FOR PREVENTING FALLS IN OLDER PEOPLE IN NURSING CARE FACILITIES AND HOSPITALS}

This review ${ }^{4}$ includes 41 randomised trials involving 25422 participants. The average age of the participants was 83 years, and around three quarters were women. Many of the participants were cognitively

School of Health and Social Care, Teesside University, Middlesbrough, UK

Correspondence to Dr H Handoll, School of Health and Social Care, Teesside University, Middlesbrough TS1 3BA, UK; h.handoll@tees.ac.uk impaired. The review reported the findings of the 30 trials conducted in nursing care facilities separately from the 11 trials in hospital settings. This reflects differences in participant characteristics between the two settings and that the differences in the environment may warrant different types of interventions, possibly implemented by people with different skill mixes. Only two trials, both in hospital settings, recruited participants with a particular health condition: respectively, stroke and hip fracture.

The review authors grouped and described interventions using the fall prevention classification system developed by the Prevention of Falls Network Europe: ProFANE. ${ }^{5}$ Where possible, the authors reported two outcomes: rate of falls ( $\mathrm{RaR}$ ) (based on the number of falls per person year) or risk of falling (RR) (based on number of trial participants who fell during follow-up). Also reported, where available, were fall related fractures. While sought, none of the included trials provided data on adverse effects of the interventions, although the possibility that some interventions increase the rate of falls was discussed in the review. None of the trials included cost effectiveness or cost utility evaluations. A summary of some of the main results of this review follows below.

Most of the trials conducted in both settings tested single interventions. Various types of supervised exercise were investigated in 11 trials in nursing care facilities, and pooled data from seven of these showed no significant difference in rate of falls or risk of falling. However, these results were inconsistent and may relate to differences among the trials in the types and intensity of exercise, the study populations, and variation in methodological quality. Probably influenced by the type of exercises, there appeared some possibility that exercise programmes for frail nursing care facility residents may increase their risk of falls. In contrast, results from three small trials conducted in subacute hospital settings showed a significant reduction in risk of falling from supervised exercise.
Pooled data from five large studies evaluating vitamin D supplementation in nursing care facilities showed a significant reduction in the rate of falls, but not risk of falling.

Pooled results from the majority of nine trials testing multifactorial interventions, which target known risk factors, in nursing care facilities did not show significantly reduced rate of falls or risk of falling. These, however, were a heterogeneous set of trials evaluating a complex intervention. A post hoc subgroup analysis pointed to a potential effect for multifactorial interventions where provided by a multidisciplinary team. There was evidence from four trials that multifactorial falls prevention programmes in hospitals for patients who have longer lengths of stay (at least 3 weeks) are effective.

Differences in study characteristics and findings for various interventions support the separate grouping of the trials by nursing care facilities and hospitals in this review. It is also pertinent to view the findings in the context of those of a related Cochrane review of interventions for preventing falls in community dwelling older people. ${ }^{6}$ Comparison of the findings of these two reviews is aided by their use of similar methodology including the using the ProFANE classification system. In contrast to the inconsistent findings for supervised exercise in nursing care facilities, a key finding of the second review is that group exercise is effective in reducing falls in older people in the community. Conversely, vitamin D was not found to be effective in reducing falls in community dwellers, although some exception was mooted for those with vitamin $\mathrm{D}$ insufficiency. Underlying vitamin $\mathrm{D}$ insufficiency may well be the basis for the protective effect found for vitamin $\mathrm{D}$ in nursing care facilities. It is noteworthy too that people with dementia were specifically excluded from the majority of the studies in the community dwellers review.

Together with those of over 4000 other Cochrane reviews, the full versions of these reviews are available in The Cochrane Library (http://www. thecochranelibrary.com). Both reviews highlighted in this article are registered with the Cochrane, Bone, Joint and Muscle Trauma Group (http://www.bjmtg. cochrane.org). The work of this group involves preparing, maintaining, and promoting the accessibility of systematic reviews on different aspects of the prevention, treatment, and rehabilitation of musculoskeletal injuries. People interested 
in contributing to this work can contact Lindsey Elstub, the Managing Editor, at lindsey.elstub@manchester.ac.uk.

\section{Competing interests None.}

Patient consent Obtained.

Provenance and peer review Not commissioned; not externally peer reviewed.

Injury Prevention 2010;16:137-138.

doi:10.1136/ip.2010.026625

\section{REFERENCES}

1. Nyberg L, Gustafson $Y$, Janson A, et al. Incidence of falls in three different types of geriatric care. A Swedish prospective study. Scand J Soc Med 1997;25:8-13.

2. Butler M, Norton R, Lee-Joe T, et al. The risks of hip fracture in older people from private homes and institutions. Age Ageing 1996;25:381-5.

3. Vassallo M, Sharma JC, Allen SC. Characteristics of single fallers and recurrent fallers among hospital in-patients. Gerontology 2002; 48:147-50.
4. Cameron ID, Murray GR, Gillespie LD, et al. Interventions for preventing falls in older people in nursing care facilities and hospitals. Cochrane Database Syst Rev 2010;(1):CD005465.

5. Lamb SE, Hauer K, Becker C. Manual for the fall prevention classification system. http://www. profane.eu.org/profane_documents/Falls_Taxonomy. pdf (accessed 29 Aprī 2009).

6. Gillespie LD, Robertson MC, Gillespie WJ, et al. Interventions for preventing falls in older people living in the community. Cochrane Database Syst Rev 2009; (2):CD007146.

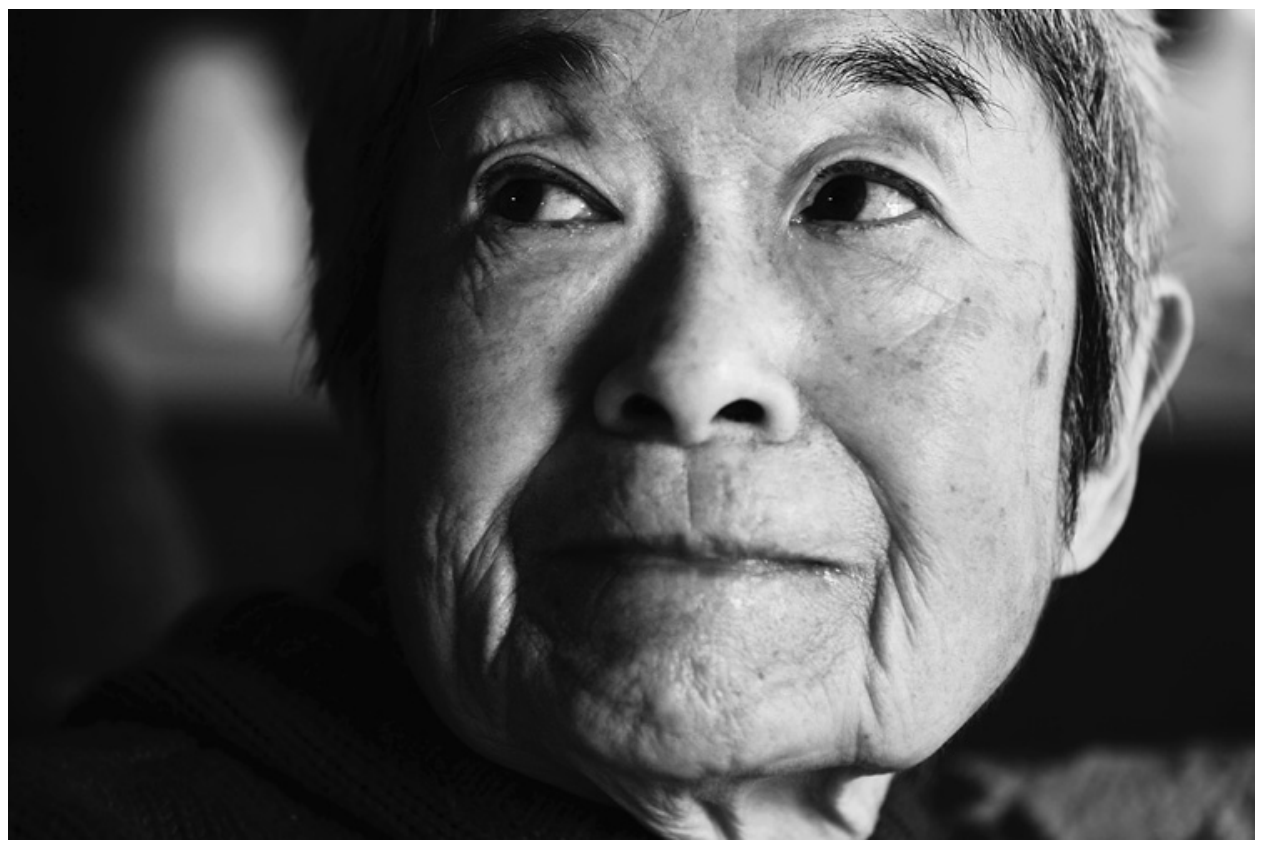

\section{Lacunae}

\section{NUDITY FORGIVEN BUT CYCLISTS FEEL HEAT OVER HELMETS}

It seems it is OK to cycle starkers in New Zealand but not without a helmet. A constable was patrolling a beach resort at night when she came across two nude men aged in their early '20s and asked them to explain. They said "they wanted to experience total freedom" the constable warned that "the way you're heading, you're going to experience total confinement" issued a warning for failing to wear helmets and sent them home with the suggestion they stay on their bikes. [From The Australian. Contributed by Ian Scott] 